\title{
CURVATURE PROPERTIES OF PSEUDO-SPHERE BUNDLES OVER PARAQUATERNIONIC MANIFOLDS
}

\author{
GABRIEL EDUARD VÎLCU, RODICA CRISTINA VOICU
}

\begin{abstract}
In this paper we obtain several curvature properties of the twistor and reflector spaces of a paraquaternionic Kähler manifold and prove the existence of both positive and negative mixed 3-Sasakian structures in a principal $S O(2,1)$-bundle over a paraquaternionic Kähler manifold.

AMS Mathematics Subject Classification: 53C26, 53C28, 53C42, 53C50. Key Words and Phrases: twistor space, Einstein manifold, paraquaternionic structure, mixed 3-structure.
\end{abstract}

\section{INTRODUCTION}

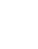

An almost paraquaternionic structure on a smooth manifold $M$ is a rank 3subbundle of $\operatorname{End}(T M)$ which is locally spanned by two almost product structures and one almost complex structure which satisfy relations of anti-commutation. Under certain conditions on the holonomy group of a metric adapted to such a structure, one arrives to the concept of paraquaternionic Kähler manifold. If $M$ is a manifold endowed with an almost paraquaternionic structure, then two kinds of unit pseudo-sphere bundle can be considered [1] the twistor space $Z^{-}(M)$ of $M$ (the unit pseudo-sphere bundle with fibre the 2-sheeted hyperboloid $S_{1}^{2}(-1)$ ) and the reflector space $Z^{+}(M)$ of $M$ (the unit pseudo-sphere bundle with fibre the 1sheeted hyperboloid $\left.S_{1}^{2}(1)\right)$. These spaces of great importance in theoretical physics (see e.g. [2, 3]) have been studied recently by several authors [4, [5, 6, 7, 8, 9, 10, We note that if $M$ is a paraquaternionic Kähler manifold, then the twistor space $Z^{-}(M)$ admits two canonical almost complex structures $I_{1}$ and $I_{2}$ ( $I_{1}$ integrable in dimension greater than $4, I_{2}$ never integrable) and the reflector space $Z^{+}(M)$ admits two canonical almost para-complex structures $P_{1}$ and $P_{2}\left(P_{1}\right.$ integrable in dimension greater than $4, P_{2}$ never integrable). Moreover, both $Z^{+}(M)$ and $Z^{-}(M)$ carries a natural 1-parameter family of pseudo-Riemannian metrics $h_{t}, t \neq 0$, such that the canonical projection $\pi_{ \pm}: Z^{ \pm}(M) \rightarrow M$ is a pseudo-Riemannian submersion [4. After giving some introductory results, in Section 2, we study the twistor and reflector spaces of a paraquaternionic Kähler manifold $M$ and the almost pseudo-Hermitian geometry of $\left(Z^{-}(M), I_{i}, h_{t}\right)$ and $\left(Z^{+}(M), P_{i}, h_{t}\right), i=1,2$. In Section 3 we give explicit formulas of the pseudo-Riemannian curvature tensors of $h_{t}$ in terms of the curvature of $M$ and study its properties in details. In particular, we determine the affiliation to Gray and para-Gray classes of $\left(Z^{-}(M), I_{i}, h_{t}\right)$ and $\left(Z^{+}(M), P_{i}, h_{t}\right), i=1,2$.

This work was partially supported by CNCSIS - UEFISCSU, project PNII - IDEI code 8/2008, contract no. 525/2009. 
On the other hand, the counterpart in odd dimension of a paraquaternionic structure was introduced in [11. It is called a mixed 3-structure, and appears in a natural way on lightlike hypersurfaces in paraquaternionic Kähler manifolds. Such hypersurfaces inherit two almost paracontact structures and an almost contact structure, satisfying analogous conditions to those satisfied by almost contact 3structures [12. This concept has been refined in [13], where the authors have introduced positive and negative metric mixed 3-structures.

W.M. Boothby and H.C. Wang gave in [14] a fibering of a compact manifold $M$ with a regular contact structure as a principal bundle over a symplectic manifold $M^{\prime}$ with 1 -dimensional toroidal group $A$. Associating a Riemannian metric $g^{\prime}$ with the symplectic structure, $M^{\prime}$ is an almost Kählerian manifold and consequently $M$ is a contact metric one. Y. Hatakeyama proved in [15] that there exists a circle bundle over an almost complex manifold that admits a normal contact metric structure. Moreover, K. Sakamoto was proved in [16] that every quaternionic Kähler manifold admits a principal bundle $P$ over it, whose structure group is $\mathrm{SO}(3)$, while Konishi [17] constructed a Sasakian 3-structure in $P$, which is canonically associated with a given quaternion Kähler structure of positive scalar curvature. It can be easily checked that the Sakamoto's construction remains valid if we consider a paraquaternionic Kähler manifold, but now the structure group is $\operatorname{SO}(2,1)$. The main purpose of the last Section is to prove that the principal bundle associated with a paraquaternionic Kähler manifold $(M, \sigma, g)$ admits both a positive and a negative mixed 3-Sasakian structure, provided that $M$ is of non-zero scalar curvature. Moreover, we investigate the existence of non-trivial Einstein metrics in the canonical variation of a pseudo-Riemannian submersion which projects a metric mixed 3-contact structure onto a paraquaternionic Kähler structure.

\section{Preliminaries}

Let $\widetilde{\mathbb{H}}$ be the algebra of paraquaternions and identify $\widetilde{\mathbb{H}}^{n}=\mathbb{R}^{4 n}$. It is well known that $\widetilde{\mathbb{H}}$ is a real Clifford algebras with $\widetilde{\mathbb{H}}=C(1,1) \cong C(0,2)$. In fact $\widetilde{\mathbb{H}}$ is generated by the unity 1 and generators $J_{1}^{0}, J_{2}^{0}, J_{3}^{0}$ satisfying the paraquaternionic identities

$$
\left(J_{1}^{0}\right)^{2}=\left(J_{2}^{0}\right)^{2}=-\left(J_{3}^{0}\right)^{2}=1, J_{1}^{0} J_{2}^{0}=-J_{2}^{0} J_{1}^{0}=J_{3}^{0} .
$$

We assume that $\widetilde{\mathbb{H}}$ acts on $\widetilde{\mathbb{H}}^{n}$ by right multiplication and use the convention that $\mathrm{SO}(2 n, 2 n)$ acts on $\widetilde{\mathbb{H}}^{n}$ on the left.

An almost product structure on a smooth manifold $M$ is a tensor field $P$ of type $(1,1)$ on $M, P \neq \pm I d$, such that $P^{2}=I d$, where $I d$ is the identity tensor field of type $(1,1)$ on $M$. The pair $(M, P)$ is called an almost product manifold. An almost para-complex manifold is an almost product manifold $(M, P)$ such that the two eigenbundles $T^{+} M$ and $T^{-} M$ associated with the two eigenvalues +1 and -1 of $P$, respectively, have the same rank. Equivalently, a splitting of the tangent bundle $T M$ of a differentiable manifold $M$, into the Whitney sum of two subbundles $T M$ of the same fiber dimension is called an almost para-complex structure on M.

An almost para-Hermitian structure on a smooth manifold $M$ is a pair $(g, P)$, where $g$ is a pseudo-Riemannian metric on $M$ and $P$ is an almost product structure on $M$, which is compatible with $g$, i.e. $P^{*} g=-g$. In this case, the triple $(M, g, P)$ is called an almost para-Hermitian manifold. Moreover, $(M, g, P)$ is said to be a para-Hermitian manifold if $P$ is integrable, i.e. if the Nijenhuis $N_{P}$ defined by

$$
N_{P}(X, Y)=[P X, P Y]-P[X, P Y]-P[P X, Y]+[X, Y]
$$


vanishes. An almost complex structure on a smooth manifold $M$ is a tensor field $J$ of type $(1,1)$ on $M$ such that $J^{2}=-I d$. The pair $(M, J)$ is called an almost complex manifold. We note that the dimension of an almost (para-)complex manifold is necessarily even (see [18, 19]).

An almost pseudo-Hermitian structure on a smooth manifold $M$ is a pair $(g, J)$, where $g$ is a pseudo-Riemannian metric on $M$ and $J$ is an almost complex structure on $M$, which is compatible with $g$, i.e. $J^{*} g=g$. In this case, the triple $(M, g, J)$ is called an almost pseudo-Hermitian manifold. Moreover, $(M, g, J)$ is said to be pseudo-Hermitian if $J$ is integrable, i.e. if the Nijenhuis $N_{J}$ defined by

$$
N_{J}(X, Y)=[J X, J Y]-J[X, J Y]-J[J X, Y]-[X, Y]
$$

vanishes.

An almost para-hypercomplex structure on a smooth manifold $M$ is a triple $H=$ $\left(J_{1}, J_{2}, J_{3}\right)$ of $(1,1)$-type tensor fields on $M$ satisfying:

$$
J_{\alpha}^{2}=-\tau_{\alpha} \mathrm{Id}, J_{\alpha} J_{\beta}=-J_{\beta} J_{\alpha}=\tau_{\gamma} J_{\gamma},
$$

for any $\alpha \in\{1,2,3\}$ and for any even permutation $(\alpha, \beta, \gamma)$ of $(1,2,3)$, where $\tau_{1}=\tau_{2}=-1=-\tau_{3}$. In this case $(M, H)$ is said to be an almost para-hypercomplex manifold. We remark that from (21) it follows that $J_{1}$ and $J_{2}$ are almost product structures on $M$, while $J_{3}=J_{1} J_{2}$ is an almost complex structure on $M$. We note that the almost para-hypercomplex structures have been introduced in geometry by P. Libermann 20] under the name of quaternionic structures of second kind (structures presque quaternioniennes de deuxième espèce).

A semi-Riemannian metric $g$ on $(M, H)$ is said to be compatible or adapted to the almost para-hypercomplex structure $H=\left(J_{\alpha}\right)_{\alpha=1,2,3}$ if it satisfies:

$$
g\left(J_{\alpha} X, J_{\alpha} Y\right)=\tau_{\alpha} g(X, Y)
$$

for all vector fields $X, Y$ on $M$ and $\alpha \in\{1,2,3\}$. Moreover, the pair $(g, H)$ is called an almost para-hyperhermitian structure on $M$ and the triple $(M, g, H)$ is said to be an almost para-hyperhermitian manifold. We note that any almost parahyperhermitian manifold is of dimension $4 m, m \geq 1$, and any adapted metric is necessarily of neutral signature $(2 m, 2 m)$. If $\left\{J_{1}, J_{2}, J_{3}\right\}$ are parallel with respect to the Levi-Civita connection of $g$, then the manifold is called para-hyper-Kähler.

An almost para-hypercomplex manifold $(M, H)$ is called a para-hypercomplex manifold if each $J_{\alpha}, \alpha=1,2,3$, is integrable. In this case $H$ is said to be a para-hypercomplex structure on $M$. Moreover, if $g$ is a semi-Riemannian metric adapted to the para-hypercomplex structure $H$, then the pair $(g, H)$ is said to be a para-hyperhermitian structure on $M$ and $(M, g, H)$ is called a para-hyperhermitian manifold.

An almost paraquaternionic Hermitian manifold is a triple $(M, \sigma, g)$, where $M$ is a smooth manifold, $\sigma$ is an almost paraquaternionic structure on $M$, i.e. a rank 3subbundle of $\operatorname{End}(T M)$ which is locally spanned by an almost para-hypercomplex structure $H=\left(J_{\alpha}\right)_{\alpha=1,2,3}$ and $g$ is a compatible metric with respect to $H$. We remark that, if $\left\{J_{1}, J_{2}, J_{3}\right\}$ and $\left\{J_{1}^{\prime}, J_{2}^{\prime}, J_{3}^{\prime}\right\}$ are two canonical local bases of $\sigma$ in $U$ and in another coordinate neighborhood $U^{\prime}$ of $M$, then we have for all $x \in U \cap U^{\prime}$

$$
\left(J_{\alpha}^{\prime}\right)_{x}=\sum_{\beta=1}^{3} s_{\alpha \beta}(x)\left(J_{\beta}^{\prime}\right)_{x}, \alpha=1,2,3,
$$


where $S_{U U^{\prime}}(x)=\left(s_{\alpha \beta}(x)\right)_{\alpha, \beta=1,2,3} \in \mathrm{SO}(2,1)$, because $\left\{J_{1}, J_{2}, J_{3}\right\}$ and $\left\{J_{1}^{\prime}, J_{2}^{\prime}, J_{3}^{\prime}\right\}$ satisfy the paraquaternionic identities (2).

If $(M, \sigma, g)$ is an almost paraquaternionic Hermitian manifold such that the bundle $\sigma$ is preserved by the Levi-Civita connection $\nabla$ of $g$, then $(M, \sigma, g)$ is said to be a paraquaternionic Kähler manifold [21]. Equivalently, we can write

$$
\nabla J_{\alpha}=\tau_{\beta} \omega_{\gamma} \otimes J_{\beta}-\tau_{\gamma} \omega_{\beta} \otimes J_{\gamma},
$$

where $(\alpha, \beta, \gamma)$ is an even permutation of $(1,2,3)$ and $\omega_{1}, \omega_{2}, \omega_{3}$ are locally defined 1 -forms. We note that the prototype of paraquaternionic Kähler manifold is the paraquaternionic projective space $P^{n}(\widetilde{\mathbb{H}})$ as described by Blažić 22 .

If the Riemannian curvature tensor $R$ is taken with the sign convention

$$
R(X, Y) Z=\nabla_{X} \nabla_{Y} Z-\nabla_{Y} \nabla_{X} Z-\nabla_{[X, Y]} Z,
$$

for all vector fields $X, Y, Z$ on $M$, then a consequence of (4) is that $R$ satisfies

$$
\left[R, J_{\alpha}\right]=\tau_{\beta} A_{\gamma} \otimes J_{\beta}-\tau_{\gamma} A_{\beta} \otimes J_{\gamma},
$$

for any even permutation $(\alpha, \beta, \gamma)$ of $(1,2,3)$, where

$$
A_{\alpha}=d \omega_{\alpha}+\tau_{\alpha} \omega_{\beta} \wedge \omega_{\gamma} .
$$

If we consider $\Omega_{\alpha}:=g\left(J_{\alpha} \cdot, \cdot\right)$ the fundamental form associated with $J_{\alpha}, \alpha=$ $1,2,3$, then we have the following structure equations (see [4, 6]):

$$
d \omega_{\alpha}+\tau_{\alpha} \omega_{\beta} \wedge \omega_{\gamma}=\tau_{\alpha} \nu \Omega_{\alpha}
$$

for any even permutation $(\alpha, \beta, \gamma)$ of $(1,2,3)$, where $\nu=\frac{S c}{4 n(n+2)}$ is the reduced scalar curvature, $S c$ being the scalar curvature defined as the trace of the Ricci tensor $\rho$.

We recall that the main property of manifolds endowed with this kind of structure is the following.

Theorem 2.1. 21] Any paraquaternionic Kähler manifold $(M, \sigma, g)$ is an Einstein space, provided that $\operatorname{dim} M>4$.

Let $(M, \sigma, g)$ be a $4 n$-dimensional paraquaternionic Kähler manifold. Then the Ricci 2-forms of the Levi-Civita connection of $g$ are defined as (see [1]):

$$
\rho_{\alpha}(X, Y)=-\frac{\tau_{\alpha}}{2} \operatorname{Trace}\left(Z \rightarrow J_{\alpha} R(X, Y) Z\right), \alpha=1,2,3,
$$

and for $n>1$ it follows

$$
\rho(X, Y)=\frac{n+2}{n} \rho_{\alpha}\left(X, J_{\alpha} Y\right), \alpha=1,2,3 .
$$

Using now Theorem 2.1 we obtain the following relations

$$
\rho_{\alpha}(X, Y)=-\tau_{\alpha} \frac{S c}{4(n+2)} g\left(X, J_{\alpha} Y\right), \alpha=1,2,3 .
$$


On the other hand, from (5) and (7) and taking account of Theorem 2.1 we obtain that the curvature tensor of a $4 n$-dimensional paraquaternionic Kähler manifold $(n>1)$, satisfies (see [21]):

$$
\begin{aligned}
& R\left(X, Y, J_{1} Z, J_{1} T\right)+R(X, Y, Z, T) \\
& =\frac{S c}{4 n(n+2)}\left\{g\left(X, J_{3} Y\right) g\left(Z, J_{3} T\right)-g\left(X, J_{2} Y\right) g\left(Z, J_{2} T\right)\right\} \\
& R\left(X, Y, J_{2} Z, J_{2} T\right)+R(X, Y, Z, T) \\
& =\frac{S c}{4 n(n+2)}\left\{g\left(X, J_{3} Y\right) g\left(Z, J_{3} T\right)-g\left(X, J_{1} Y\right) g\left(Z, J_{1} T\right)\right\} \\
& R\left(X, Y, J_{3} Z, J_{3} T\right)-R(X, Y, Z, T) \\
& =\frac{S c}{4 n(n+2)}\left\{g\left(X, J_{2} Y\right) g\left(Z, J_{2} T\right)+g\left(X, J_{1} Y\right) g\left(Z, J_{1} T\right)\right\}
\end{aligned}
$$

for all vector fields $X, Y, Z$ and $T$ on $M$, where $R(X, Y, Z, T)=g(R(X, Y) Z, T)$.

We consider now the general case of a $4 n$-dimensional smooth manifold $M$ endowed with an almost paraquaternionic structure $\sigma$ and with a paraquaternionic connection $\nabla$, i.e. a linear connection which preserves $\sigma$, and following [1] we recall some basic facts concerning the twistor and reflector spaces of $M$, which will be useful in the next Section.

Let $p \in M$. Any linear frame $u$ on $T_{p} M$ can be considered as an isomorphism $u: \mathbb{R}^{4 n} \rightarrow T_{p} M$. Taking such a frame $u$ we can define a subspace of of the space of the all endomorphisms of $T_{p} M$ by $u(\operatorname{sp}(1, \mathbb{R})) u^{-1}$. This subset is a paraquaternionic structure and we define $P(M)$ to be the set of all linear frames $u$ which satisfy $u(\operatorname{sp}(1, \mathbb{R})) u^{-1}=\sigma$, where $\operatorname{sp}(1, \mathbb{R})=\operatorname{Span}\left\{J_{1}^{0}, J_{2}^{0}, J_{3}^{0}\right\}$ is the Lie algebra of $\operatorname{Sp}(1, \mathbb{R})$. It is clear that $P(M)$ is the principal frame bundle of $M$ with structure group $\operatorname{GL}(n, \widetilde{\mathbb{H}}) \operatorname{Sp}(1, \mathbb{R})$, where

$$
\operatorname{GL}(n, \widetilde{\mathbb{H}})=\left\{A \in \mathrm{GL}(4 n, \mathbb{R}) \mid A(\operatorname{sp}(1, \mathbb{R})) A^{-1}=\operatorname{sp}(1, \mathbb{R})\right\} .
$$

We denote by $\pi: P(M) \rightarrow M$ the natural projection and remark that the Lie algebra of $\mathrm{GL}(n, \widetilde{\mathbb{H}})$ is

$$
\operatorname{gl}(n, \widetilde{\mathbb{H}})=\{A \in \operatorname{gl}(4 n, \mathbb{R}) \mid A B=B A \text { for all } B \in \operatorname{sp}(1, \mathbb{R})\} .
$$

We also denote by $(, \quad)$ the inner product in $\operatorname{gl}(4 n, \mathbb{R})$ given by $(A, B)=$ $\operatorname{Trace}\left(A B^{t}\right)$, for $A, B \in \operatorname{gl}(4 n, \mathbb{R})$.

We split now the curvature of $\nabla$ into $\operatorname{gl}(n, \widetilde{\mathbb{H}})$-valued part $R^{\prime}$ and $\operatorname{sp}(1, \mathbb{R})$-valued part $R^{\prime \prime}$ following the classical scheme (see e.g. [23]). We denote the splitting of the $\operatorname{gl}(n, \widetilde{\mathbb{H}}) \oplus \operatorname{sp}(1, \mathbb{R})$-valued curvature 2 -form $\Omega$ on $P(M)$ according to the splitting of the curvature $R$, by

$$
\Omega=\Omega^{\prime}+\Omega^{\prime \prime},
$$

where $\Omega^{\prime}$ is a $\operatorname{gl}(n, \widetilde{\mathbb{H}})$-valued 2 -form and $\Omega^{\prime \prime}$ is a $\operatorname{sp}(1, \mathbb{R})$-valued form. Explicitly,

$$
\Omega^{\prime \prime}=\Omega_{1}^{\prime \prime} J_{1}^{0}+\Omega_{2}^{\prime \prime} J_{2}^{0}+\Omega_{3}^{\prime \prime} J_{3}^{0},
$$

where $\Omega_{\alpha}^{\prime \prime}, \alpha=1,2,3$ are 2 -forms. If $\xi, \eta \in \mathbb{R}^{4 n}$, then the 2 -forms $\Omega_{\alpha}^{\prime \prime}, \alpha=1,2,3$, are given by

$$
\Omega_{\alpha}^{\prime \prime}(B(\xi), B(\eta))=\frac{1}{2 n} \rho_{\alpha}(X, Y)=-\tau_{\alpha} \frac{S c}{8 n(n+2)} g\left(X, J_{\alpha} Y\right)
$$


where $X=u(\xi), Y=u(\eta)$ (see [1]).

For each $u \in P(M)$ we consider two linear isomorphism $j^{+}(u)$ and $j^{-}(u)$ on $T_{\pi(u)} M$ defined by:

$$
j^{+}(u)=u J_{1}^{0} u^{-1}, \quad j^{-}(u)=u J_{3}^{0} u^{-1} .
$$

It is easy to see that

$$
\left(j^{-}(u)\right)^{2}=-I d, \quad\left(j^{+}(u)\right)^{2}=I d
$$

and

$$
g\left(j^{-}(u) X, j^{-}(u) Y\right)=g(X, Y), \quad g\left(j^{+}(u) X, j^{+}(u) Y\right)=-g(X, Y),
$$

for all $X, Y \in T_{\pi(u)} M$.

As in [1, for each $p \in M$ we consider

$$
Z_{p}^{ \pm}(M)=\left\{j^{ \pm}(u) \mid u \in P(M), \pi(u)=p\right\}
$$

and we define the twistor space $Z^{-}$of $M$ and the reflector space $Z^{+}$of $M$, by setting

$$
Z^{ \pm}=Z^{ \pm}(M)=\bigcup_{p \in M} Z_{p}^{ \pm}(M)
$$

Then the twistor space $Z^{-}(M)$ is the unit pseudo-sphere bundle with fibre the 2 -sheeted hyperboloid $S_{1}^{2}(-1)=\left\{(x, y, z) \in \mathbb{R}^{3} \mid x^{2}+y^{2}-z^{2}=-1\right\}$ and the reflector space $Z^{+}(M)$ is the unit pseudo-sphere bundle with fibre the 1-sheeted hyperboloid $S_{1}^{2}(1)=\left\{(x, y, z) \in \mathbb{R}^{3} \mid x^{2}+y^{2}-z^{2}=1\right\}$.

We denote by $A^{*}$ (resp. $B(\xi)$ ) the fundamental vector field (resp. the standard horizontal vector field) on $P(M)$, the principal frame bundle of $M$, corresponding to $A \in \operatorname{gl}(n, \widetilde{\mathbb{H}}) \oplus \operatorname{sp}(1, \mathbb{R})$ (resp. $\left.\xi \in \mathbb{R}^{4 n}\right)$. Let $u \in P(M)$ and $Q_{u}$ be the horizontal subspace of the tangent space $T_{u} P(M)$ induced by the connection $\nabla$ on $M$ (see [19]). As in [1] we have the decompositions

$$
T_{u} P(M)=\left(h_{i}\right)_{u}^{*} \oplus\left(m_{i}\right)_{u}^{*} \oplus Q_{u}, i=1,3
$$

and the following isomorphisms

where

$$
\begin{gathered}
\left.j_{* u}^{-}\right|_{\left(m_{3}\right)_{u}^{*} \oplus Q_{u}}:\left(m_{3}\right)_{u}^{*} \oplus Q_{u} \rightarrow T_{j^{-}(u)} Z^{-}, \\
\left.j_{* u}^{+}\right|_{\left(m_{1}\right)_{u}^{*} \oplus Q_{u}}:\left(m_{1}\right)_{u}^{*} \oplus Q_{u} \rightarrow T_{j^{+}(u)} Z^{+}
\end{gathered}
$$

$$
\begin{gathered}
h_{3}=\left\{A \in \operatorname{gl}(n, \widetilde{\mathbb{H}}) \oplus \operatorname{sp}(1, \mathbb{R}) \mid A J_{3}^{0}=J_{3}^{0} A\right\}, \\
h_{1}=\left\{A \in \operatorname{gl}(n, \widetilde{\mathbb{H}}) \oplus \operatorname{sp}(1, \mathbb{R}) \mid A J_{1}^{0}=J_{1}^{0} A\right\}, \\
m_{3}=\left\{A \in \operatorname{gl}(n, \widetilde{\mathbb{H}}) \oplus \operatorname{sp}(1, \mathbb{R}) \mid A J_{3}^{0}=-J_{3}^{0} A\right\}=\operatorname{Span}\left\{J_{1}^{0}, J_{2}^{0}\right\}, \\
m_{1}=\left\{A \in \operatorname{gl}(n, \widetilde{\mathbb{H}}) \oplus \operatorname{sp}(1, \mathbb{R}) \mid A J_{1}^{0}=-J_{1}^{0} A\right\}=\operatorname{Span}\left\{J_{2}^{0}, J_{3}^{0}\right\},
\end{gathered}
$$

and

$$
\left(h_{i}\right)_{u}^{*}=\left\{A_{u}^{*} \mid A \in h_{i}\right\},\left(m_{i}\right)_{u}^{*}=\left\{A_{u}^{*} \mid A \in m_{i}\right\}, i=1,3 .
$$

Now, we can define two almost complex structures $I_{1}$ and $I_{2}$ on $Z^{-}$by (see [1])

$$
\begin{aligned}
& I_{1}\left(j_{* u}^{-} A^{*}\right)=j_{* u}^{-}\left(J_{3}^{0} A\right)^{*}, \\
& I_{2}\left(j_{* u}^{-} A^{*}\right)=-j_{* u}^{-}\left(J_{3}^{0} A\right)^{*} \\
& I_{i}\left(j_{* u}^{-} B(\xi)\right)=j_{* u}^{-} B\left(J_{3}^{0} \xi\right), i=1,2,
\end{aligned}
$$

for $u \in P(M), A \in m_{3}, \xi \in \mathbb{R}^{4 n}$. 
Similarly, it can be defined two almost paracomplex structures $P_{1}$ and $P_{2}$ on $Z^{+}$ by (see [1])

$$
\begin{aligned}
& P_{1}\left(j_{* u}^{+} A^{*}\right)=j_{* u}^{+}\left(J_{1}^{0} A\right)^{*}, \\
& P_{2}\left(j_{* u}^{+} A^{*}\right)=-j_{* u}^{+}\left(J_{1}^{0} A\right)^{*} \\
& P_{i}\left(j_{* u}^{+} B(\xi)\right)=j_{* u}^{+} B\left(J_{1}^{0} \xi\right), i=1,2,
\end{aligned}
$$

for $u \in P(M), A \in m_{1}, \xi \in \mathbb{R}^{4 n}$.

We remark that the almost complex structures defined above were also defined and investigated in [6] on paraquaternionic Kähler manifolds, the authors proving that the almost complex structure $I_{2}$ is never integrable while $I_{1}$ is always integrable. Moreover, we note that in [1] the authors found that the para-complex structures $P_{2}$ is never integrable on reflector space, while $P_{1}$ is always integrable.

\section{Curvature Properties of $\left(Z^{-}, I_{i}, h_{t}\right)$ AND $\left(Z^{+}, P_{i}, h_{t}\right), i=1,2$ ON PARAQUATERNIONIC KÄHLER MANIFOLDS}

Let $(M, \sigma, g)$ be a $4 n$-dimensional paraquaternionic Kähler manifold. Since the Levi-Civita connection $\nabla$ of $g$ is a paraquaternionic connection on $M$, it follows that all the considerations in the last part of the above sections remain valid if the manifold is endowed with a paraquaternionic Kähler structure. Then, as in 4, 6, 9, we can define a natural 1-parameter family of pseudo-Riemannian metrics $h_{t}, t \neq 0$, on $Z^{-}$by

$$
\begin{gathered}
h_{t}\left(j_{* u}^{-} A^{*}, j_{* u}^{-} B^{*}\right)=t(A, B), \\
h_{t}\left(j_{* u}^{-} A^{*}, j_{* u}^{-} B(\xi)\right)=0, \\
h_{t}\left(j_{* u}^{-} B(\xi), j_{* u}^{-} B(\eta)\right)=g(X, Y),
\end{gathered}
$$

for $u \in P(M), A, B \in m_{3}, \xi, \eta \in \mathbb{R}^{4 n}$ and $X=u(\xi), Y=u(\eta)$, and similarly on $Z^{+}$:

$$
\begin{gathered}
h_{t}\left(j_{* u}^{+} A^{*}, j_{* u}^{+} B^{*}\right)=t(A, B), \\
h_{t}\left(j_{* u}^{+} A^{*}, j_{* u}^{+} B(\xi)\right)=0, \\
h_{t}\left(j_{* u}^{+} B(\xi), j_{* u}^{+} B(\eta)\right)=g(X, Y),
\end{gathered}
$$

for $u \in P(M), A, B \in m_{1}, \xi, \eta \in \mathbb{R}^{4 n}$ and $X=u(\xi), Y=u(\eta)$.

It is easy now to see that $\left(I_{i}, h_{t}\right), i=1,2$ (resp. $\left.\left(P_{i}, h_{t}\right), i=1,2\right)$ determine two families of almost pseudo-Hermitian structures on $Z^{-}$(resp. two families of almost para-Hermitian structures on $Z^{+}$).

The computations performed in 24] for the metric twistor space over Riemannian manifold $M$ can be adapted in our cases, using (15) and (17) for the twistor space and respectively (16) and (18) for the reflector space. We obtain for the curvature tensors $K^{ \pm}$, where $K^{-}$is the curvature tensor on the twistor space and $K^{+}$is the 
curvature tensor on the reflector space, the following expressions:

$$
\begin{gathered}
K^{ \pm}\left(j_{* u}^{ \pm} A^{*}, j_{* u}^{ \pm} B^{*}, j_{* u}^{ \pm} C^{*}, j_{* u}^{ \pm} D^{*}\right)=-t([A, B],[C, D]) \\
K^{ \pm}\left(j_{* u}^{ \pm} A^{*}, j_{* u}^{ \pm} B^{*}, j_{* u}^{ \pm} C^{*}, j_{* u}^{ \pm} B(\xi)\right)=0 \\
K^{ \pm}\left(j_{* u}^{ \pm} A^{*}, j_{* u}^{ \pm} B(\xi), j_{* u}^{ \pm} B^{*}, j_{* u}^{ \pm} B(\eta)\right)=\frac{t}{2}([A, B], \Omega(B(\xi), B(\eta))(u))- \\
-\frac{t^{2}}{4} \varepsilon_{i}\left(B, \Omega\left(B(\xi), B\left(e_{i}\right)\right)(u)\right)\left(A, \Omega\left(B(\eta), B\left(e_{i}\right)\right)(u)\right) \\
K^{ \pm}\left(j_{* u}^{ \pm} B(\xi), j_{* u}^{ \pm} B(\eta), j_{* u}^{ \pm} B(\zeta), j_{* u}^{ \pm} A^{*}\right)=\frac{t}{2}\left(A, B(\zeta)_{u}(\Omega(B(\xi), B(\eta)))\right) \\
K^{ \pm}\left(j_{* u}^{ \pm} B(\xi), j_{* u}^{ \pm} B(\eta), j_{* u}^{ \pm} B(\zeta), j_{* u}^{ \pm} B(\tau)\right)=R(u(\xi), u(\eta), u(\zeta), u(\tau))- \\
-\frac{t}{4}\left\{\left(\Omega_{m_{ \pm}}(B(\eta), B(\zeta))(u), \Omega_{m_{ \pm}}(B(\xi), B(\tau))(u)\right)-\right. \\
-\left(\Omega_{m_{ \pm}}(B(\xi), B(\zeta))(u), \Omega_{m_{ \pm}}(B(\eta), B(\tau))(u)\right)- \\
\left.-2\left(\Omega_{m_{ \pm}}(B(\xi), B(\eta))(u), \Omega_{m_{ \pm}}(B(\zeta), B(\tau))(u)\right)\right\}
\end{gathered}
$$

for $u \in P(M), A, B, C, D \in m_{ \pm}$, where $m_{-}=m_{3}, m_{+}=m_{1}, \xi, \eta, \zeta, \tau \in \mathbb{R}^{4 n}$ and $\Omega_{m_{ \pm}}$denotes the $m_{ \pm}$-component of $\Omega$.

Using now (10)-(14) in (19) we derive

$$
\begin{gathered}
K^{-}\left(j_{* u}^{-} A^{*}, j_{* u}^{-} B^{*}, j_{* u}^{-} C^{*}, j_{* u}^{-} D^{*}\right)=\frac{t}{n}\left(J_{3}^{0} A, B\right)\left(J_{3}^{0} C, D\right) \\
K^{-}\left(j_{* u}^{-} A^{*}, j_{* u}^{-} B^{*}, j_{* u}^{-} C^{*}, j_{* u}^{-} B(\xi)\right)=0 \\
K^{-}\left(j_{* u}^{-} A^{*}, j_{* u}^{-} B(\xi), j_{* u}^{-} B^{*}, j_{* u}^{-} B(\eta)\right)= \\
=\left(-\frac{t^{2} S c^{2}}{64 n(n+2)^{2}}+\frac{t S c}{8 n(n+2)}\right)\left(J_{3}^{0} A, B\right) g\left(J_{3} X, Y\right)+\frac{t^{2} S c^{2}}{64 n(n+2)^{2}}(A, B) g(X, Y) \\
K^{-}\left(j_{* u}^{-} B(\xi), j_{* u}^{-} B(\eta), j_{* u}^{-} B(\zeta), j_{* u}^{-} A^{*}\right)=0 \\
K^{-}\left(j_{* u}^{-} B(\xi), j_{* u}^{-} B(\eta), j_{* u}^{-} B(\zeta), j_{* u}^{-} B(\tau)\right)=R(X, Y, Z, T)- \\
-\frac{t S c^{2}}{64 n(n+2)^{2}}\left\{g\left(Y, J_{1} Z\right) g\left(X, J_{1} T\right)+g\left(Y, J_{2} Z\right) g\left(X, J_{2} T\right)-\right. \\
-g\left(X, J_{1} Z\right) g\left(Y, J_{1} T\right)-g\left(X, J_{2} Z\right) g\left(Y, J_{2} T\right)- \\
\left.-2 g\left(X, J_{1} Y\right) g\left(Z, J_{1} T\right)-2 g\left(X, J_{2} Y\right) g\left(Z, J_{2} T\right)\right\}
\end{gathered}
$$

for $u \in P(M), A, B, C, D \in m_{3}, \xi, \eta, \zeta, \tau \in \mathbb{R}^{4 n}$ and $u(\xi)=X, u(\eta)=Y, u(\zeta)=$ $Z, u(\tau)=T$ and

$$
\begin{gathered}
K^{+}\left(j_{* u}^{+} A^{*}, j_{* u}^{+} B^{*}, j_{* u}^{+} C^{*}, j_{* u}^{+} D^{*}\right)=\frac{t}{n}\left(J_{1}^{0} A, B\right)\left(J_{1}^{0} C, D\right) \\
K^{+}\left(j_{* u}^{+} A^{*}, j_{* u}^{+} B^{*}, j_{* u}^{+} C^{*}, j_{* u}^{+} B(\xi)\right)=0 \\
K^{+}\left(j_{* u}^{+} A^{*}, j_{* u}^{+} B(\xi), j_{* u}^{+} B^{*}, j_{* u}^{+} B(\eta)\right)= \\
(21)=\left(-\frac{t^{2} S c^{2}}{64 n(n+2)^{2}}-\frac{t S c}{8 n(n+2)}\right)\left(J_{1}^{0} A, B\right) g\left(J_{1} X, Y\right)-\frac{t^{2} S c^{2}}{64 n(n+2)^{2}}(A, B) g(X, Y) \\
K^{+}\left(j_{* u}^{+} B(\xi), j_{* u}^{+} B(\eta), j_{* u}^{+} B(\zeta), j_{* u}^{+} A^{*}\right)=0 \\
K^{+}\left(j_{* u}^{+} B(\xi), j_{* u}^{+} B(\eta), j_{* u}^{+} B(\zeta), j_{* u}^{+} B(\tau)\right)=R(X, Y, Z, T)- \\
-\frac{t S c^{2}}{64 n(n+2)^{2}}\left\{-g\left(Y, J_{2} Z\right) g\left(X, J_{2} T\right)+g\left(Y, J_{3} Z\right) g\left(X, J_{3} T\right)+\right. \\
+g\left(X, J_{2} Z\right) g\left(Y, J_{2} T\right)-g\left(X, J_{3} Z\right) g\left(Y, J_{3} T\right)+ \\
\left.+2 g\left(X, J_{2} Y\right) g\left(Z, J_{2} T\right)-2 g\left(X, J_{3} Y\right) g\left(Z, J_{3} T\right)\right\}
\end{gathered}
$$

for $u \in P(M), A, B, C, D \in m_{1}, \xi, \eta, \zeta, \tau \in \mathbb{R}^{4 n}$ and $u(\xi)=X, u(\eta)=Y, u(\zeta)=$ $Z, u(\tau)=T$. 
We recall now that the $*$-Ricci tensor of a $2 n$-dimensional almost pseudo-Hermitian manifold $(M, g, J)$ is defined by

$$
\rho^{*}(X, Y)=\sum_{i=1}^{2 n} \varepsilon_{i} R\left(X, E_{i}, J Y, J E_{i}\right)
$$

where $R$ denotes the curvature of the metric $g,\left\{E_{1}, \ldots, E_{2 n}\right\}$ is a pseudo-orthonormal basis at an arbitrary point $p$ and $X, Y$ are tangent vectors at $p$. If the ${ }^{*}$-Ricci tensor is scalar multiple of the metric then the manifold is said to be ${ }^{*}$-Einstein.

On the other hand, we note that A. Gray introduced in [25] three basic classes $A H 1, A H 2, A H 3$ of almost Hermitian manifolds, whose curvature tensors resemble that of a Kähler manifold. They are defined by the following curvature identities:

$$
\begin{gathered}
A H 1: R(X, Y, Z, T)=R(X, Y, J Z, J T), \\
A H 2: R(X, Y, Z, T)=R(J X, J Y, Z, T)+R(J X, Y, J Z, T)+R(J X, Y, Z, J T), \\
A H 3: R(X, Y, Z, T)=R(J X, J Y, J Z, J T),
\end{gathered}
$$

where $R$ is the curvature tensor of the manifold. It is easy to see that

$$
A H 1 \subset A H 2 \subset A H 3 .
$$

By analogy, one says that an almost para-Hermitian manifold satisfies the paraGray identities if

$$
\begin{gathered}
A P H 1: R(X, Y, Z, T)=-R(X, Y, J Z, J T), \\
A P H 2: R(X, Y, Z, T)=-R(J X, J Y, Z, T)-R(J X, Y, J Z, T)-R(J X, Y, Z, J T), \\
A P H 3: R(X, Y, Z, T)=R(J X, J Y, J Z, J T),
\end{gathered}
$$

where $R$ is the curvature tensor of the manifold. We note that para-Gray-like identities were considered in [26, 27]) and it can be easily checked that

$$
A P H 1 \subset A P H 2 \subset A P H 3 .
$$

We can give now the main curvature properties of $\left(Z^{-}, I_{i}, h_{t}\right)$ and $\left(Z^{+}, P_{i}, h_{t}\right)$, $i=1,2$, in the following two theorems.

Theorem 3.1. Let $(M, \sigma, g)$ be a $4 n$-dimensional paraquaternionic Kähler manifold and $\left(Z^{-}, I_{i}, h_{t}\right), i=1,2$ the twistor spaces associated. Then:

(i) The manifolds $\left(Z^{-}, I_{i}, h_{t}\right), i=1,2$, belong always to $A H 2$ and $A H 3$ and are with pseudo-Hermitian Ricci tensor and with pseudo-Hermitian *-Ricci tensor;

(ii) The manifolds $\left(Z^{-}, I_{1}, h_{t}\right)$ belong to $A H 1$ iff $S c=0$ or $S c=\frac{4(n+2)}{t}$;

(iii) The manifolds $\left(Z^{-}, I_{2}, h_{t}\right)$ belong to $A H 1$ iff $S c=0$;

(iv) The manifolds $\left(Z^{-}, I_{i}, h_{t}\right), i=1,2$ are Einstein iff

$$
S c=\frac{4(n+2)}{t} \text { or } S c=\frac{4(n+2)}{(n+1) t}
$$

(v) The manifolds $\left(Z^{-}, I_{1}, h_{t}\right)$ are ${ }^{*}$-Einstein iff

$$
S c=\frac{4(n+2)}{t} \text { or } S c=-\frac{4(n+2)}{n t} \text {; }
$$

(vi) The manifolds $\left(Z^{-}, I_{2}, h_{t}\right)$ are ${ }^{*}$-Einstein iff

$$
S c=\frac{2(n+2)}{(n-1) t}\left[3 n-1-\sqrt{9 n^{2}-10 n+5}\right]
$$


or

$$
S c=\frac{2(n+2)}{(n-1) t}\left[3 n-1+\sqrt{9 n^{2}-10 n+5}\right] .
$$

Proof. (i) This statement follows from (1), (9), (13), (15) and (20) after some long but straightforward computations.

$($ ii $)+($ iii $)$ From (15) and (20) we obtain

$$
\begin{gathered}
K^{-}\left(j_{* u}^{-} A^{*}, j_{* u}^{-} B^{*}, I_{i}\left(j_{* u}^{-} C^{*}\right), I_{i}\left(j_{* u}^{-} D^{*}\right)\right)=\frac{t}{n}\left(J_{3}^{0} A, B\right)\left(J_{3}^{0} C, D\right) \\
K^{-}\left(j_{* u}^{-} A^{*}, j_{* u}^{-} B^{*}, I_{i}\left(j_{* u}^{-} C^{*}\right), I_{i}\left(j_{* u}^{-} B(\xi)\right)\right)=0 \\
K^{-}\left(j_{* u}^{-} A^{*}, j_{* u}^{-} B(\xi), I_{i}\left(j_{* u}^{-} B^{*}\right), I_{i}\left(j_{* u}^{-} B(\eta)\right)\right)= \\
=\left(\varepsilon_{i} \frac{t^{2} S c^{2}}{64 n(n+2)^{2}}-\varepsilon_{i} \frac{t S c}{8 n(n+2)}\right)(A, B) g(X, Y)-\varepsilon_{i} \frac{t^{2} S c^{2}}{64 n(n+2)^{2}}\left(J_{3}^{0} A, B\right) g\left(J_{3} X, Y\right) \\
K^{-}\left(j_{* u}^{-} B(\xi), j_{* u}^{-} B(\eta), I_{i}\left(j_{* u}^{-} B(\zeta)\right), I_{i}\left(j_{* u}^{-} A^{*}\right)\right)=0 \\
K^{-}\left(j_{* u}^{-} B(\xi), j_{* u}^{-} B(\eta), I_{i}\left(j_{* u}^{-} B(\zeta)\right), I_{i}\left(j_{* u}^{-} B(\tau)\right)\right)=R(X, Y, Z, T)- \\
-\frac{t S c^{2}}{64 n(n+2)^{2}}\left\{g\left(Y, J_{1} Z\right) g\left(X, J_{1} T\right)+g\left(Y, J_{2} Z\right) g\left(X, J_{2} T\right)-\right. \\
\left.-g\left(X, J_{1} Z\right) g\left(Y, J_{1} T\right)-g\left(X, J_{2} Z\right) g\left(Y, J_{2} T\right)\right\}+ \\
+\left(\frac{S c}{4 n(n+2)}-\frac{2 t S c^{2}}{64 n(n+2)^{2}}\right)\left(g\left(X, J_{1} Y\right) g\left(Z, J_{1} T\right)+g\left(X, J_{2} Y\right) g\left(Z, J_{2} T\right)\right)
\end{gathered}
$$

for $i=1,2, \varepsilon_{1}=-1, \varepsilon_{2}=+1, u \in P(M), A, B, C, D \in m_{3}, \xi, \eta, \zeta, \tau \in \mathbb{R}^{4 n}$ and $u(\xi)=X, u(\eta)=Y, u(\zeta)=Z, u(\tau)=T$. Now the conclusion follows from (20) and (22).

$(i v)+(v)+(v i)$ Using (20) we obtain the following formulas for the Ricci tensors $\rho^{-}$of $\left(Z^{-}, h_{t}\right)$ :

$$
\begin{gathered}
\rho^{-}\left(j_{* u}^{-} A^{*}, j_{* u}^{-} B^{*}\right)=\left[\frac{t S c^{2}}{16(n+2)^{2}}+\frac{1}{n t}\right] h_{t}\left(j_{* u}^{-} A^{*}, j_{* u}^{-} B^{*}\right) \\
\rho^{-}\left(j_{* u}^{-} A^{*}, j_{* u}^{-} B(\xi)\right)=0 \\
\rho^{-}\left(j_{* u}^{-} B(\xi), j_{* u}^{-} B(\eta)\right)=\left[\frac{S c}{4 n}-\frac{t S c^{2}}{16 n(n+2)^{2}}\right] h_{t}\left(j_{* u}^{-} B(\xi), j_{* u}^{-} B(\eta)\right)
\end{gathered}
$$

Similarly, from (15) and (20) we get ${ }^{*}$-Ricci tensors $\rho_{1}^{*-}$ of $\left(Z^{-}, I_{1}, h_{t}\right), \rho_{2}^{*-}$ of $\left(Z^{-}, I_{2}, h_{t}\right)$ :

$$
\begin{gathered}
\rho_{1}^{*-}\left(j_{* u}^{-} A^{*}, j_{* u}^{-} B^{*}\right)=\left[-\frac{t S c^{2}}{16(n+2)^{2}}+\frac{S c}{2(n+2)}+\frac{1}{n t}\right] h_{t}\left(j_{* u}^{-} A^{*}, j_{* u}^{-} B^{*}\right) \\
\rho_{2}^{*-}\left(j_{* u}^{-} A^{*}, j_{* u}^{-} B^{*}\right)=\left[\frac{t S c^{2}}{16(n+2)^{2}}-\frac{S c}{2(n+2)}+\frac{1}{n t}\right] h_{t}\left(j_{* u}^{-} A^{*}, j_{* u}^{-} B^{*}\right) \\
\rho_{1}^{*-}\left(j_{* u}^{-} A^{*}, j_{* u}^{-} B(\xi)\right)=\rho_{2}^{*-}\left(j_{* u}^{-} A^{*}, j_{* u}^{-} B(\xi)\right)=0 \\
\rho_{1}^{*-}\left(j_{* u}^{-} B(\xi), j_{* u}^{-} B(\eta)\right)=\left[\frac{S c(n+1)}{4 n(n+2)}\right] h_{t}\left(j_{* u}^{-} B(\xi), j_{* u}^{-} B(\eta)\right) \\
\rho_{2}^{*-}\left(j_{* u}^{-} B(\xi), j_{* u}^{-} B(\eta)\right)=\left[\frac{t S c^{2}}{16 n(n+2)^{2}}+\frac{S c(n-1)}{4 n(n+2)}\right] h_{t}\left(j_{* u}^{-} B(\xi), j_{* u}^{-} B(\eta)\right)
\end{gathered}
$$

Now $(i v),(v)$ and $(v i)$ follows from (23) and (24).

Theorem 3.2. Let $(M, \sigma, g)$ be a $4 n$-dimensional paraquaternionic Kähler manifold and $\left(Z^{+}, P_{i}, h_{t}\right), i=1,2$, the reflector spaces associated. Then:

(i) The manifolds $\left(Z^{+}, P_{i}, h_{t}\right), i=1,2$, belong always to APH2 and APH3 and are with para-Hermitian Ricci tensor and with para-Hermitian ${ }^{*}$-Ricci tensor;

(ii) The manifolds $\left(Z^{+}, P_{1}, h_{t}\right)$ belong to $A P H 1$ iff $S c=0$ or $S c=-\frac{4(n+2)}{t}$; 
(iii) The manifolds $\left(Z^{+}, P_{2}, h_{t}\right)$ belong to APH1 iff $S c=0$;

(iv) The manifolds $\left(Z^{+}, P_{i}, h_{t}\right), i=1,2$ are Einstein iff

$$
S c=-\frac{4(n+2)}{t} \text { or } S c=-\frac{4(n+2)}{(n+1) t}
$$

(v) The manifolds $\left(Z^{+}, P_{1}, h_{t}\right)$ are ${ }^{*}$-Einstein iff

$$
S c=-\frac{4(n+2)}{t} \text { or } S c=\frac{4(n+2)}{n t}
$$

(vi) The manifolds $\left(Z^{+}, P_{2}, h_{t}\right)$ are ${ }^{*}$-Einstein iff

$$
S c=\frac{2(n+2)}{(n-1) t}\left[1-3 n-\sqrt{9 n^{2}-10 n+5}\right]
$$

or

$$
S c=\frac{2(n+2)}{(n-1) t}\left[1-3 n+\sqrt{9 n^{2}-10 n+5}\right] .
$$

Proof. (i) This statement follows from (11), (9), (14), (16) and (21) after some long but straightforward computations.

$($ ii $)+($ iii $)$ From (16) and (21) we obtain

$$
\begin{gathered}
K^{+}\left(j_{* u}^{+} A^{*}, j_{* u}^{+} B^{*}, P_{i}\left(j_{* u}^{+} C^{*}\right), P_{i}\left(j_{* u}^{+} D^{*}\right)\right)=-\frac{t}{n}\left(J_{1}^{0} A, B\right)\left(J_{1}^{0} C, D\right) \\
K^{+}\left(j_{* u}^{+} A^{*}, j_{* u}^{+} B^{*}, P_{i}\left(j_{* u}^{+} C^{*}\right), P_{i}\left(j_{* u}^{+} B(\xi)\right)\right)=0 \\
K^{+}\left(j_{* u}^{+} A^{*}, j_{* u}^{+} B(\xi), P_{i}\left(j_{* u}^{+} B^{*}\right), P_{i}\left(j_{* u}^{+} B(\eta)\right)\right)= \\
(25)=\left(\varepsilon_{i} \frac{t^{2} S c^{2}}{64 n(n+2)^{2}}+\varepsilon_{i} \frac{t S c}{8 n(n+2)}\right)(A, B) g(X, Y)+\varepsilon_{i} \frac{t^{2} S c^{2}}{64 n(n+2)^{2}}\left(J_{1}^{0} A, B\right) g\left(J_{1} X, Y\right) \\
K^{+}\left(j_{* u}^{+} B(\xi), j_{* u}^{+} B(\eta), P_{i}\left(j_{* u}^{+} B(\zeta)\right), P_{i}\left(j_{* u}^{+} A^{*}\right)\right)=0 \\
K^{+}\left(j_{* u}^{+} B(\xi), j_{* u}^{+} B(\eta), P_{i}\left(j_{* u}^{+} B(\zeta)\right), P_{i}\left(j_{* u}^{+} B(\tau)\right)\right)=-R(X, Y, Z, T)- \\
-\frac{t S c^{2}}{64 n(n+2)^{2}}\left\{g\left(Y, J_{2} Z\right) g\left(X, J_{2} T\right)-g\left(Y, J_{3} Z\right) g\left(X, J_{3} T\right)-\right. \\
\left.-g\left(X, J_{2} Z\right) g\left(Y, J_{2} T\right)+g\left(X, J_{3} Z\right) g\left(Y, J_{3} T\right)\right\}+ \\
+\left(\frac{S c}{4 n(n+2)}+\frac{2 t S c^{2}}{64 n(n+2)^{2}}\right)\left(-g\left(X, J_{2} Y\right) g\left(Z, J_{2} T\right)+g\left(X, J_{3} Y\right) g\left(Z, J_{3} T\right)\right)
\end{gathered}
$$

for $i=1,2, \varepsilon_{1}=-1, \varepsilon_{2}=+1, u \in P(M), A, B, C, D \in m_{1}, \xi, \eta, \zeta, \tau \in \mathbb{R}^{4 n}$ and $u(\xi)=X, u(\eta)=Y, u(\zeta)=Z, u(\tau)=T$.

Now the conclusion follows from (21) and (25).

$(i v)+(v)+(v i)$ Using (21) we obtain the following formulas for the Ricci tensors $\rho^{+}$of $\left(Z^{+}, h_{t}\right)$ :

$$
\begin{gathered}
\rho^{+}\left(j_{* u}^{+} A^{*}, j_{* u}^{+} B^{*}\right)=\left[-\frac{t S c^{2}}{16(n+2)^{2}}-\frac{1}{n t}\right] h_{t}\left(j_{* u}^{+} A^{*}, j_{* u}^{+} B^{*}\right) \\
\rho^{+}\left(j_{* u}^{+} A^{*}, j_{* u}^{+} B(\xi)\right)=0 \\
\rho^{+}\left(j_{* u}^{+} B(\xi), j_{* u}^{+} B(\eta)\right)=\left[\frac{S c}{4 n}+\frac{t S c^{2}}{16 n(n+2)^{2}}\right] h_{t}\left(j_{* u}^{+} B(\xi), j_{* u}^{+} B(\eta)\right) .
\end{gathered}
$$

Similarly, from (16) and (21) we get ${ }^{*}$-Ricci tensors $\rho_{1}^{*+}$ of $\left(Z^{+}, P_{1}, h_{t}\right)$ and $\rho_{2}^{*+}$ of $\left(Z^{+}, P_{2}, h_{t}\right)$ : 


$$
\begin{gathered}
\rho_{1}^{*+}\left(j_{* u}^{+} A^{*}, j_{* u}^{+} B^{*}\right)=\left[-\frac{t S c^{2}}{16(n+2)^{2}}-\frac{S c}{2(n+2)}+\frac{1}{n t}\right] h_{t}\left(j_{* u}^{+} A^{*}, j_{* u}^{+} B^{*}\right) \\
\rho_{2}^{*+}\left(j_{* u}^{+} A^{*}, j_{* u}^{+} B^{*}\right)=\left[\frac{t S c^{2}}{16(n+2)^{2}}+\frac{S c}{2(n+2)}+\frac{1}{n t}\right] h_{t}\left(j_{* u}^{+} A^{*}, j_{* u}^{+} B^{*}\right) \\
\rho_{1}^{*+}\left(j_{* u}^{+} A^{*}, j_{* u}^{+} B(\xi)\right)=\rho_{2}^{*+}\left(j_{* u}^{+} A^{*}, j_{* u}^{+} B(\xi)\right)=0 \\
\rho_{1}^{*+}\left(j_{* u}^{+} B(\xi), j_{* u}^{+} B(\eta)\right)=\left[-\frac{S c(n+1)}{4 n(n+2)}\right] h_{t}\left(j_{* u}^{+} B(\xi), j_{* u}^{+} B(\eta)\right) \\
\rho_{2}^{*+}\left(j_{* u}^{+} B(\xi), j_{* u}^{+} B(\eta)\right)=\left[\frac{t S c^{2}}{16 n(n+2)^{2}}-\frac{S c(n-1)}{4 n(n+2)}\right] h_{t}\left(j_{* u}^{+} B(\xi), j_{* u}^{+} B(\eta)\right) .
\end{gathered}
$$

Now (iv), (v) and (vi) follows from (26) and (27).

Remark 3.3. We note that the corresponding result of the Theorem 3.1 in quaternionic setting was obtained in 28 and the assertions stated at items (iv) in Theorems 3.1 and 3.2 were also proved, but using a different method, by Alekseevsky and Cortés (compare with 4, Corollary 6, page 126]).

\section{Mixed 3-Sasakian structures in a SO(2,1)-Principal Bundle over a PARAQUATERNIONIC KÄHLER MANIFOLD}

Definition 4.1. Let $M$ be a smooth manifold equipped with a triple $(\varphi, \xi, \eta)$, where $\varphi$ is a field of endomorphisms of the tangent spaces, $\xi$ is a vector field and $\eta$ is a 1 -form on $M$. If we have:

$$
\varphi^{2}=\tau(-I+\eta \otimes \xi), \quad \eta(\xi)=1
$$

then we say that:

(i) $(\varphi, \xi, \eta)$ is an almost contact structure on $M$, if $\tau=1$ (29]).

(ii) $(\varphi, \xi, \eta)$ is an almost paracontact structure on $M$, if $\tau=-1$ (30]).

We remark that many authors also include in the above definition the conditions that

$$
\varphi \xi=0, \eta \circ \varphi=0,
$$

although these are deducible from (28) (see [31).

Definition 4.2. [13] A mixed 3-structure on a smooth manifold $M$ is a triple of structures $\left(\varphi_{\alpha}, \xi_{\alpha}, \eta_{\alpha}\right), \alpha \in\{1,2,3\}$, which are almost paracontact structures for $\alpha=1,2$ and almost contact structure for $\alpha=3$, satisfying the following conditions:

$$
\begin{gathered}
\eta_{\alpha}\left(\xi_{\beta}\right)=0 \\
\varphi_{\alpha}\left(\xi_{\beta}\right)=\tau_{\beta} \xi_{\gamma}, \quad \varphi_{\beta}\left(\xi_{\alpha}\right)=-\tau_{\alpha} \xi_{\gamma}, \\
\eta_{\alpha} \circ \varphi_{\beta}=-\eta_{\beta} \circ \varphi_{\alpha}=\tau_{\gamma} \eta_{\gamma}, \\
\varphi_{\alpha} \varphi_{\beta}-\tau_{\alpha} \eta_{\beta} \otimes \xi_{\alpha}=-\varphi_{\beta} \varphi_{\alpha}+\tau_{\beta} \eta_{\alpha} \otimes \xi_{\beta}=\tau_{\gamma} \varphi_{\gamma},
\end{gathered}
$$

where $(\alpha, \beta, \gamma)$ is an even permutation of $(1,2,3)$ and $\tau_{1}=\tau_{2}=-\tau_{3}=-1$.

Moreover, if a manifold $M$ with a mixed 3-structure $\left(\varphi_{\alpha}, \xi_{\alpha}, \eta_{\alpha}\right)_{\alpha=\overline{1,3}}$ admits a semi-Riemannian metric $g$ such that:

$$
g\left(\varphi_{\alpha} X, \varphi_{\alpha} Y\right)=\tau_{\alpha}\left[g(X, Y)-\varepsilon_{\alpha} \eta_{\alpha}(X) \eta_{\alpha}(Y)\right],
$$

for all $X, Y \in \Gamma(T M)$ and $\alpha=1,2,3$, where $\varepsilon_{\alpha}=g\left(\xi_{\alpha}, \xi_{\alpha}\right)= \pm 1$, then we say that $M$ has a metric mixed 3-structure and $g$ is called a compatible metric. 
Remark 4.3. From (33) we obtain

$$
\eta_{\alpha}(X)=\varepsilon_{\alpha} g\left(X, \xi_{\alpha}\right), g\left(\varphi_{\alpha} X, Y\right)=-g\left(X, \varphi_{\alpha} Y\right)
$$

for all $X, Y \in \Gamma(T M)$ and $\alpha=1,2,3$.

Note that if $\left(M,\left(\varphi_{\alpha}, \xi_{\alpha}, \eta_{\alpha}\right)_{\alpha=\overline{1,3}}, g\right)$ is a manifold with a metric mixed 3-structure then from (34) it follows

$$
g\left(\xi_{1}, \xi_{1}\right)=g\left(\xi_{2}, \xi_{2}\right)=-g\left(\xi_{3}, \xi_{3}\right) .
$$

Hence the vector fields $\xi_{1}$ and $\xi_{2}$ are both either space-like or time-like and these force the causal character of the third vector field $\xi_{3}$. We may therefore distinguish between positive and negative metric mixed 3-structures, according as $\xi_{1}$ and $\xi_{2}$ are both space-like, or both time-like vector fields. Because one can check that, at each point of $M$, there always exists a pseudo-orthonormal frame field given by $\left\{\left(E_{i}, \varphi_{1} E_{i}, \varphi_{2} E_{i}, \varphi_{3} E_{i}\right)_{i=\overline{1, n}}, \xi_{1}, \xi_{2}, \xi_{3}\right\}$ we conclude that the dimension of the manifold is $4 n+3$ and the signature of $g$ is $(2 n+1,2 n+2)$, where we put first the minus signs, if the metric mixed 3 -structure is positive (i.e. $\varepsilon_{1}=\varepsilon_{2}=-\varepsilon_{3}=1$ ), or the signature of $g$ is $(2 n+2,2 n+1)$, if the metric mixed 3 -structure is negative (i.e. $\varepsilon_{1}=\varepsilon_{2}=-\varepsilon_{3}=-1$ ).

Definition 4.4. 13] Let $M$ be a manifold endowed with a (positive/negative) metric mixed 3-structure $\left(\left(\varphi_{\alpha}, \xi_{\alpha}, \eta_{\alpha}\right)_{\alpha=\overline{1,3}}, g\right)$. This structure is said to be:

(i) a (positive/negative) metric mixed 3-contact structure if $d \eta_{\alpha}=\Phi_{\alpha}$, for each $\alpha \in\{1,2,3\}$, where $\Phi_{\alpha}$ is the fundamental 2-form defined by $\Phi_{\alpha}(X, Y):=$ $g\left(X, \varphi_{\alpha} Y\right)$.

(ii) a (positive/negative) mixed 3-K-contact structure if $\left(\left(\varphi_{3}, \xi_{3}, \eta_{3}\right), g\right)$ is a $\mathrm{K}$ contact structure and $\left(\left(\varphi_{1}, \xi_{1}, \eta_{1}\right), g\right),\left(\left(\varphi_{2}, \xi_{2}, \eta_{2}\right), g\right)$ are para-K-contact structures, i.e. $\nabla \xi_{\alpha}=-\varepsilon_{\alpha} \varphi_{\alpha}$, for each $\alpha \in\{1,2,3\}$, where $\nabla$ is Levi-Civita connection of $g$.

(iii) a (positive/negative) mixed 3-Sasakian structure if $\left(\varphi_{3}, \xi_{3}, \eta_{3}, g\right)$ is a Sasakian structure and $\left(\varphi_{1}, \xi_{1}, \eta_{1}, g\right),\left(\varphi_{2}, \xi_{2}, \eta_{2}, g\right)$ are para-Sasakian structures, i.e.

$$
\left(\nabla_{X} \varphi_{\alpha}\right) Y=\tau_{\alpha}\left[g(X, Y) \xi_{\alpha}-\varepsilon_{\alpha} \eta_{\alpha}(Y) X\right]
$$

for all $X, Y \in \Gamma(T M)$ and $\alpha \in\{1,2,3\}$.

Remark 4.5. Note that in fact mixed metric 3-contact, mixed 3-K-contact and mixed 3-Sasakian structures define the same class of manifolds, as proved in [13. Moreover, like their Riemannian counterparts, mixed 3-Sasakian structures are Einstein, but now the scalar curvature can be either positive or negative:

Theorem 4.6. [13, 32] Any $(4 n+3)$-dimensional manifold endowed with a mixed 3 -Sasakian structure is an Einstein space with Einstein constant $\lambda=(4 n+2) \varepsilon$, with $\varepsilon=\mp 1$, according as the metric mixed 3-structure is positive or negative, respectively.

Remark 4.7. Several examples of manifolds endowed with mixed 3-Sasakian structures can be found in 33. We note that the unit pseudo-sphere $S_{2 n+2}^{4 n+3} \subset \mathbb{R}_{2 n+2}^{4 n+4}$ is the canonical example of manifold with negative mixed 3-Sasakian structure, while the pseudo-hyperbolic space $H_{2 n+1}^{4 n+3} \subset \mathbb{R}_{2 n+2}^{4 n+4}$ can be endowed with a canonical positive mixed 3-Sasakian structure.

Let $(M, \sigma, g)$ be a paraquaternionic Kähler manifold and let $P$ be the bundle associated with $(M, \sigma, g)$. That is, $P$ is the bundle whose transition functions and 
structure group are the same as $\sigma$, but whose fibre is $\mathrm{SO}(2,1)$. The Lie algebra of $\mathrm{SO}(2,1)$ is $\operatorname{so}(2,1)$ and we consider two of its bases: $\mathcal{B}^{+}=\left\{e_{1}, e_{2}, e_{3}\right\}$ and $\mathcal{B}^{-}=\left\{-e_{1},-e_{2},-e_{3}\right\}$, where

$$
e_{1}=\left(\begin{array}{lll}
0 & 0 & 0 \\
0 & 0 & 2 \\
0 & 2 & 0
\end{array}\right), e_{2}=\left(\begin{array}{lll}
0 & 0 & 2 \\
0 & 0 & 0 \\
2 & 0 & 0
\end{array}\right), e_{3}=\left(\begin{array}{ccc}
0 & -2 & 0 \\
2 & 0 & 0 \\
0 & 0 & 0
\end{array}\right) .
$$

It can be easily verified that

$$
\left[e_{1}, e_{2}\right]=2 e_{3},\left[e_{2}, e_{3}\right]=-2 e_{1},\left[e_{3}, e_{1}\right]=-2 e_{2},
$$

and

$$
\left[-e_{1},-e_{2}\right]=-2\left(-e_{3}\right),\left[-e_{2},-e_{3}\right]=2\left(-e_{1}\right),\left[-e_{3},-e_{1}\right]=2\left(-e_{2}\right) .
$$

On the other hand, their exp are

$$
\begin{aligned}
\exp \left(t e_{1}\right) & =\left(\begin{array}{ccc}
1 & 0 & 0 \\
0 & \cosh 2 t & \sinh 2 t \\
0 & \sinh 2 t & \cosh 2 t
\end{array}\right), \\
\exp \left(t e_{2}\right) & =\left(\begin{array}{ccc}
\cosh 2 t & 0 & \sinh 2 t \\
0 & 1 & 0 \\
\sinh 2 t & 0 & \cosh 2 t
\end{array}\right), \\
\exp \left(t e_{2}\right) & =\left(\begin{array}{ccc}
\cos 2 t & -\sin 2 t & 0 \\
\sin 2 t & \cos 2 t & 0 \\
0 & 0 & 1
\end{array}\right) .
\end{aligned}
$$

Then, with respect to the basis $\mathcal{B}^{+}$, we have

$$
\operatorname{ad}\left(e_{1}\right)=\left(\begin{array}{lll}
0 & 0 & 0 \\
0 & 0 & 2 \\
0 & 2 & 0
\end{array}\right), \operatorname{ad}\left(e_{2}\right)=\left(\begin{array}{ccc}
0 & 0 & -2 \\
0 & 0 & 0 \\
-2 & 0 & 0
\end{array}\right), \operatorname{ad}\left(e_{3}\right)=\left(\begin{array}{ccc}
0 & 2 & 0 \\
-2 & 0 & 0 \\
0 & 0 & 0
\end{array}\right)
$$

and with respect to the basis $\mathcal{B}^{-}$, we have

$$
\operatorname{ad}\left(-e_{1}\right)=\left(\begin{array}{ccc}
0 & 0 & 0 \\
0 & 0 & -2 \\
0 & -2 & 0
\end{array}\right), \operatorname{ad}\left(-e_{2}\right)=\left(\begin{array}{lll}
0 & 0 & 2 \\
0 & 0 & 0 \\
2 & 0 & 0
\end{array}\right), \operatorname{ad}\left(-e_{3}\right)=\left(\begin{array}{ccc}
0 & -2 & 0 \\
2 & 0 & 0 \\
0 & 0 & 0
\end{array}\right) .
$$

For each neighborhood $U$ of $(M, \sigma, g)$, we define a so(2,1)-valued 1-form on $U$ by

$$
\omega_{U}=\sum_{i=1}^{3} \omega_{i} e_{i} .
$$

From (3) and (38) we obtain that on $U \cap U^{\prime}$ we have

$$
\omega_{U^{\prime}}(X)=\operatorname{ad}\left(S_{U U^{\prime}}^{-1}\right) \cdot \omega_{U}(X)+\left(S_{U U^{\prime}}\right)_{*}(X) \cdot S_{U U^{\prime}}^{-1}
$$

for all vector fields $X$ on $P$, where $\left(S_{U U^{\prime}}\right)_{*}$ denotes the differential of the mapping $S_{U U^{\prime}}: U \cap U^{\prime} \rightarrow \mathrm{SO}(2,1)$. Therefore there exists a connection form $\omega$ on $P$ such that

$$
\psi^{*} \omega=\omega_{U},
$$

where $\psi$ is a certain local cross-section of $P$ over $U$. 
The curvature form $\Omega$ defined by the connection $\omega$ is a so(2,1)-valued 2 -form given by

$$
\Omega(X, Y)=d \omega(X, Y)+\frac{1}{2}[\omega(X), \omega(Y)]
$$

for all vector fields $X, Y$ on $P$. Hence, using (36) we derive:

$$
\psi^{*} \Omega=\left(d \omega_{1}-\omega_{2} \wedge \omega_{3}\right) e_{1}+\left(d \omega_{2}-\omega_{3} \wedge \omega_{1}\right) e_{2}+\left(d \omega_{3}+\omega_{1} \wedge \omega_{2}\right) e_{3} .
$$

Theorem 4.8. The principal bundle $P$ associated with a paraquaternionic Kähler manifold $(M, \sigma, g)$ of non-zero scalar curvature can be endowed with both a positive and a negative mixed 3-Sasakian structure.

Proof. If $\omega_{U}=\sum_{i=1}^{3} \omega_{i} e_{i}$ is the infinitesimal connection in $P$ considered above, then we can define a semi-Riemannian metric $\widetilde{g}$ on $P$ by

$$
\widetilde{g}=\nu \pi_{*} g+\sum_{i=1}^{3} \varepsilon_{i} \omega_{i} \otimes \omega_{i}
$$

where $\nu$ is the reduced scalar curvature of $M$ and $\left(\varepsilon_{1}, \varepsilon_{2}, \varepsilon_{3}\right)=(-1,-1,1)$ (Case I) or $\left(\varepsilon_{1}, \varepsilon_{2}, \varepsilon_{3}\right)=(1,1,-1)$ (Case II).

We remark that unlike quaternionic setting (see [17]), the signature of $\widetilde{g}$ does not depend on the sign of the scalar curvature. In fact, the signature is $(2 n+2,2 n+1)$ in the Case I and $(2 n+1,2 n+2)$ in the Case II.

Firstly, we consider the Case I. We define the 1-forms $\eta_{\alpha}=\omega_{\alpha}$ for $\alpha=1,2,3$, and let $\xi_{1}, \xi_{2}, \xi_{3}$ be the fundamental vector fields corresponding to $e_{1}, e_{2}, e_{3}$, respectively. Then from (36) we deduce

$$
\left[\xi_{1}, \xi_{2}\right]=2 \xi_{3},\left[\xi_{2}, \xi_{3}\right]=-2 \xi_{1},\left[\xi_{3}, \xi_{1}\right]=-2 \xi_{2}
$$

and

$$
\eta_{\alpha}\left(\xi_{\beta}\right)=\delta_{\alpha \beta}, \alpha, \beta=1,2,3 .
$$

If $\widetilde{\nabla}$ is the Levi-Civita connection of $\widetilde{g}$, then we define

$$
\varphi_{\alpha}=-\varepsilon_{\alpha} \widetilde{\nabla} \xi_{\alpha}, \alpha=1,2,3
$$

Next we prove that $\left(\left(\varphi_{\alpha}, \xi_{\alpha}, \eta_{\alpha}\right)_{\alpha=\overline{1,3}}, \widetilde{g}\right)$ is a negative mixed 3-Sasakian structure on $P$.

If $p \in P$, then we denote by $T_{p}^{V}(P)$ the tangent space of a fibre at $p$ and by $T_{p}^{H}(P)$ its orthogonal complemented space in $T_{p} P$. Using now (41) and Koszul formula, we derive

$$
\varphi_{\alpha}\left(\xi_{\alpha}\right)=0, \varphi_{\alpha}\left(\xi_{\beta}\right)=\tau_{\beta} \xi_{\gamma}, \quad \varphi_{\beta}\left(\xi_{\alpha}\right)=-\tau_{\alpha} \xi_{\gamma} .
$$

From (42) it follows that $T_{p}^{V}(P)$ and $T_{p}^{H}(P)$ are invariant under the action of $\varphi_{\alpha}, \alpha=1,2,3$. Therefore we have:

$$
\varphi_{\alpha}=\varphi_{\alpha}^{H}+\tau_{\beta} \eta_{\beta} \otimes \xi_{\gamma}-\tau_{\gamma} \eta_{\gamma} \otimes \xi_{\beta}
$$

for any even permutation $(\alpha, \beta, \gamma)$ of $(1,2,3)$, where $\varphi_{\alpha}^{H}$ denotes the restricted actions of $\varphi_{\alpha}$ on $T_{p}^{H}(P)$, for $\alpha=1,2,3$.

Now, from the structure equations (6), we obtain for each neighborhood $U$ in $M$ and a local cross section $\psi: U \rightarrow P$ the following relation

$$
\left(d \omega_{\alpha}+\tau_{\alpha} \omega_{\beta} \wedge \omega_{\gamma}\right)\left(\psi_{*} X, \psi_{*} Y\right)=\tau_{\alpha} \nu g\left(J_{\alpha} X, Y\right),
$$


for any even permutation $(\alpha, \beta, \gamma)$ of $(1,2,3)$, where $\psi_{*}$ denotes the differential of $\psi$. Since the curvature form is horizontal, we have

$$
\left(\varphi_{\alpha}-\tau_{\beta} \eta_{\beta} \otimes \xi_{\gamma}+\tau_{\gamma} \eta_{\gamma} \otimes \xi_{\beta}\right)\left(\psi_{*} X\right)=\left(\psi_{*} J_{\alpha} X\right)^{H},
$$

so we derive

$$
\varphi_{\alpha}\left(\psi_{*} X\right)=\left(\psi_{*} J_{\alpha} X\right)^{H}+\tau_{\beta} \eta_{\beta}\left(\psi_{*} X\right) \xi_{\gamma}-\tau_{\gamma} \eta_{\gamma}\left(\psi_{*} X\right) \xi_{\beta}
$$

Using now (2), (40) and (44) we obtain

$$
\begin{aligned}
\varphi_{\alpha}^{2}\left(\psi_{*} X\right) & =\varphi_{\alpha}\left[\left(\psi_{*} J_{\alpha} X\right)^{H}+\tau_{\beta} \eta_{\beta}\left(\psi_{*} X\right) \xi_{\gamma}-\tau_{\gamma} \eta_{\gamma}\left(\psi_{*} X\right) \xi_{\beta}\right] \\
& =\left(\psi_{*} J_{\alpha}^{2} X\right)^{H}-\tau_{\beta} \tau_{\gamma} \eta_{\gamma}\left(\psi_{*} X\right) \xi_{\gamma}-\tau_{\gamma} \tau_{\beta} \eta_{\beta}\left(\psi_{*} X\right) \xi_{\beta} \\
& =-\tau_{\alpha}\left(\psi_{*} X\right)^{H}-\tau_{\beta} \tau_{\gamma}\left[\eta_{\gamma}\left(\psi_{*} X\right) \xi_{\gamma}+\eta_{\beta}\left(\psi_{*} X\right) \xi_{\beta}\right]
\end{aligned}
$$

and since $\tau_{\alpha} \tau_{\beta} \tau_{\gamma}=1$, we deduce

$$
\begin{aligned}
\varphi_{\alpha}^{2}\left(\psi_{*} X\right) & =-\tau_{\alpha}\left[\left(\psi_{*} X\right)^{H}+\eta_{\gamma}\left(\psi_{*} X\right) \xi_{\gamma}+\eta_{\beta}\left(\psi_{*} X\right) \xi_{\beta}\right] \\
& =-\tau_{\alpha}\left[\psi_{*} X-\eta_{\alpha}\left(\psi_{*} X\right) \xi_{\alpha}\right] .
\end{aligned}
$$

From (40) and (45) we conclude that $\left(\varphi_{1}, \xi_{1}, \eta_{1}\right)$ and $\left(\varphi_{2}, \xi_{2}, \eta_{2}\right)$ are almost paracontact structures on $P$, while $\left(\varphi_{3}, \xi_{3}, \eta_{3}\right)$ is an almost contact structure on $P$. Now we are able to prove the compatibility conditions between these structures.

Using again (21), (40) and (44) we obtain

$$
\begin{aligned}
\varphi_{\alpha} \varphi_{\beta}\left(\psi_{*} X\right) & =\varphi_{\alpha}\left[\left(\psi_{*} J_{\beta} X\right)^{H}+\tau_{\gamma} \eta_{\gamma}\left(\psi_{*} X\right) \xi_{\alpha}-\tau_{\alpha} \eta_{\alpha}\left(\psi_{*} X\right) \xi_{\gamma}\right] \\
& =\left(\psi_{*} J_{\alpha} J_{\beta} X\right)^{H}+\tau_{\alpha} \tau_{\gamma} \eta_{\alpha}\left(\psi_{*} X\right) \xi_{\beta} \\
& =\tau_{\gamma}\left[\left(\psi_{*} J_{\gamma} X\right)^{H}+\tau_{\alpha} \eta_{\alpha}\left(\psi_{*} X\right) \xi_{\beta}\right] \\
& =\tau_{\gamma}\left[\varphi_{\gamma}\left(\psi_{*} X\right)+\tau_{\beta} \eta_{\beta}\left(\psi_{*} X\right) \xi_{\alpha}\right]
\end{aligned}
$$

and taking into account that $\tau_{\alpha} \tau_{\beta} \tau_{\gamma}=1$, we deduce

$$
\varphi_{\alpha} \varphi_{\beta}\left(\psi_{*} X\right)=\tau_{\gamma} \varphi_{\gamma}\left(\psi_{*} X\right)+\tau_{\alpha}\left(\psi_{*} X\right) \xi_{\alpha} .
$$

Similarly we find

$$
\varphi_{\beta} \varphi_{\alpha}\left(\psi_{*} X\right)=-\tau_{\gamma} \varphi_{\gamma}\left(\psi_{*} X\right)+\tau_{\beta}\left(\psi_{*} X\right) \xi_{\beta} .
$$

and so the compatibility condition (32) is verified. Analogously we find that the compatibility condition (31) is checked. Moreover, from (40) and (42) we have that (29) and (30) are also true.

On the other hand, using (39) and (44) we derive

$$
\begin{aligned}
\widetilde{g}\left(\varphi_{\alpha} \psi_{*} X, \varphi_{\alpha} \psi_{*} Y\right) & =\nu g\left(J_{\alpha} X, J_{\alpha} Y\right)+\varepsilon_{\beta} \eta_{\gamma}\left(\psi_{*} X\right) \eta_{\gamma}\left(\psi_{*} Y\right)+\varepsilon_{\gamma} \eta_{\beta}\left(\psi_{*} X\right) \eta_{\beta}\left(\psi_{*} Y\right) \\
& =\tau_{\alpha}\left[\nu g(X, Y)+\varepsilon_{\beta} \eta_{\beta}\left(\psi_{*} X\right) \eta_{\beta}\left(\psi_{*} Y\right)+\varepsilon_{\gamma} \eta_{\gamma}\left(\psi_{*} X\right) \eta_{\gamma}\left(\psi_{*} Y\right)\right] \\
& =\tau_{\alpha}\left[\widetilde{g}\left(\psi_{*} X, \psi_{*} Y\right)-\varepsilon_{\alpha} \eta_{\alpha}\left(\psi_{*} X\right) \eta_{\alpha}\left(\psi_{*} Y\right)\right] .
\end{aligned}
$$

Hence the metric $\widetilde{g}$ is compatible with $\left(\varphi_{\alpha}, \xi_{\alpha}, \eta_{\alpha}\right)$, for $\alpha=1,2,3$.

Therefore, taking account of (41), we deduce that $\left(\left(\varphi_{\alpha}, \xi_{\alpha}, \eta_{\alpha}\right)_{\alpha=\overline{1,3}}, \widetilde{g}\right)$ is a negative mixed 3-K-contact structure in $P$, and so, via Remark 4.5, is a negative mixed 3-Sasakian structure in $P$.

In the Case II, we consider $\xi_{1}^{\prime}, \xi_{2}^{\prime}, \xi_{3}^{\prime}$ to be the fundamental vector fields corresponding to $-e_{1},-e_{2},-e_{3}$, respectively, and we define

$$
\eta_{\alpha}^{\prime}=-\omega_{\alpha}, \varphi_{\alpha}^{\prime}=-\varepsilon_{\alpha} \widetilde{\nabla} \xi_{\alpha}^{\prime}
$$

for $\alpha=1,2,3$. Then, it can be proved similarly as in Case I that $\left(\left(\varphi_{\alpha}^{\prime}, \xi_{\alpha}^{\prime}, \eta_{\alpha}^{\prime}\right)_{\alpha=\overline{1,3}}, \widetilde{g}\right)$ is a positive mixed 3 -Sasakian structure in $P$. 
Remark 4.9. We note that in [34, the authors investigated the possible projectability onto paraquaternionic structures of metric mixed 3-contact structures defined on the total space of a $G$-principal bundle. In fact they proved that if $P(M, G, \pi)$ is a $G$-principal bundle, where the total space $P$ is endowed with a metric mixed 3-contact structure $\left(\left(\varphi_{\alpha}, \xi_{\alpha}, \eta_{\alpha}\right)_{\alpha=\overline{1,3}}, g\right), G$ is a Lie group acting on the right on $P$ by isometries having Lie algebra isomorphic to so $(2,1)$ and such that the vector fields $\xi_{1}, \xi_{2}, \xi_{3}$ are fundamental vector fields, then the metric mixed 3-contact structure projects via $\pi$ onto a paraquaternionic Kähler structure $(\sigma, h)$ on the base space $M$ and $\pi:(P, g) \rightarrow(M, h)$ is a pseudo-Riemannian submersion. Moreover, if the dimension of $P$ is $(4 n+3)$, then one has

$$
\rho^{M}=\varepsilon 4(n+2) h
$$

were $\rho^{M}$ denotes the Ricci curvature tensor of $M$ and $\varepsilon=\mp 1$, according as the mixed 3-structure is positive or negative.

Let now $X, Y$ and $U, V$ be basic and vertical vector fields throughout. The metric $g$ splits up as

$$
g(E, F)=g(X+U, Y+V)=g(X, Y)+g(U, V),
$$

where $E=X+U, F=Y+V$ are vector fields on $P$. Then the canonical variation of the metric $g$ for $t>0$, is a pseudo-Riemannian metric $g_{t}$ on $P$ defined by (see [35])

$$
g_{t}(X+U, Y+V)=g(X, Y)+t g(U, V) .
$$

Similarly as in the Riemannian case [36, it can be proved that the curvature tensor $R_{t}$ of the canonical variation $g_{t}$ satisfies

$$
\begin{aligned}
R_{t}(E, F, G, H)= & t R(E, F, G, H)+(1-t) R^{N}\left(X_{*}, Y_{*}, Z_{*}, Z_{*}^{\prime}\right)+ \\
& +t(1-t)\left(g\left(S_{E} H, S_{F} G\right)-g\left(S_{E} G, S_{F} H\right)\right)
\end{aligned}
$$

where $E, F, G, H$ are vector fields on $P, X_{*}, Y_{*}, Z_{*}, Z_{*}^{\prime}$ are the corresponding vector fields on $M$ of the basic vector fields $X, Y, Z, Z^{\prime}$ and $S_{E} F=T_{U} V+A_{X} V+A_{Y} U$, $E=X+U, F=Y+V, G=Z+W, H=Z^{\prime}+W^{\prime}, T$ and $A$ being the O'Neill tensors of the submersion (see [37]). In this setting, we can state now the following

Theorem 4.10. If $\pi:\left(P,\left(\varphi_{\alpha}, \xi_{\alpha}, \eta_{\alpha}\right)_{\alpha=\overline{1,3}}, g\right) \rightarrow(M, \sigma, h)$ is a pseudo-Riemannian submersion as in the previous remark, then there exist a unique positive $t \neq 1$ such that $g_{t}$ is also Einstein.

Proof. We denote by $R$ the curvature tensor of manifold $P$ and by $\rho, \rho^{M}$ the Ricci curvature tensor of manifold $P$, respectively $M$. Using (34) and (35) we obtain that

$$
R(E, F) \xi_{\alpha}=\tau_{\alpha}\left(\eta_{\alpha}(F) E-\eta_{\alpha}(E) F\right), \alpha=1,2,3,
$$

where $E, F$ are vector fields on $P$.

Now using (47), (48), (49) and the definitions of mixed 3-Sasakian and paraquaternionic Kähler structures we obtain that the components of the Ricci tensor $\rho_{t}$ of the canonical variation $g_{t}$ are given by

$$
\begin{aligned}
& \rho_{t}(U, V)=t \rho(U, V)+\left\{-4 n \varepsilon t^{2}+(4 n \varepsilon+2 \varepsilon) t-2 \varepsilon\right\} g(U, V), \\
& \rho_{t}(U, X)=t \rho(U, X) \\
& \rho_{t}(X, Y)=t \rho(X, Y)+(1-t) \rho^{M}\left(X_{*}, Y_{*}\right)
\end{aligned}
$$


where $X, Y$ and $U, V$ are basic and vertical vector fields on $P, X_{*}, Y_{*}$ are the vector fields on $M$ corresponding to the basic vector fields $X, Y, \varepsilon=\mp 1$ according as the metric mixed 3-structure is positive or negative, respectively.

From Theorem 2.1 Theorem 4.6 and (46) we derive

$$
\begin{aligned}
& \rho_{t}(U, V)=\left\{-4 n \varepsilon t^{2}+(8 n \varepsilon+4 \varepsilon) t-2 \varepsilon\right\} g(U, V), \\
& \rho_{t}(U, X)=0, \\
& \rho_{t}(X, Y)=\{-6 \varepsilon t+4 n \varepsilon+8 \varepsilon\} g(X, Y),
\end{aligned}
$$

Now, from (50) we deduce easily that $g_{t}$ is Einstein if and only if $t=1$ or $t=\frac{2 n+5}{2 n}$ and the conclusion follows.

\section{REFERENCES}

[1] S. Ivanov, I. Minchev and S. Zamkovoy, Twistor and reflector spaces of almost paraquaternionic manifolds, Cortés, Vicente (ed.), Handbook of pseudo-Riemannian geometry and supersymmetry, IRMA Lectures in Mathematics and Theoretical Physics 16 (2010), 477-496.

[2] R. Penrose, The central programme of twistor theory, Chaos, Solitons and Fractals 10 (1999), 581-611.

[3] E. Witten, Perturbative gauge theory as a string theory in twistor space, Commun. Math. Phys. 252 (2004), 189-258.

[4] D. Alekseevsky and V. Cortés, The twistor spaces of a para-quaternionic Kähler manifold, Osaka J. Math. 45 (2008), no. 1, 215-251.

[5] D.V. Alekseevsky, C. Medori and A. Tomassini, Homogeneous para-Kähler Einstein manifolds, Russ. Math. Surv. 64 (2009), no. 1, 1-43.

[6] D.E. Blair, J. Davidov and O. Muškarov, Hyperbolic twistor spaces, Rocky Mt. J. Math. 35 (2005), no. 5, 1437-1465.

[7] A.S. Dancer, H.R. Jørgensen and A.F. Swann, Metric geometries over the split quaternions, Rend. Semin. Mat., Torino 63 (2005), no. 2, 119-139.

[8] L. David, About the geometry of almost para-quaternionic manifolds, Diff. Geom. Applic. 27 (2009), 575-588.

[9] S. Ivanov and S. Zamkovoy, Parahermitian and paraquaternionic manifolds, Differ. Geom. Appl. 23 (2005), 205-234.

[10] L. Schäfer, On the structure of nearly pseudo-Kähler manifolds, Monatsh. Math. 163(3) (2011), 339-371.

[11] S. Ianuş, R. Mazzocco and G.E. Vîlcu, Real lightlike hypersurfaces of paraquaternionic Kähler manifolds, Mediterr. J. Math. 3 (2006), 581-592.

[12] Y. Kuo, On almost contact 3-structure, Tohoku Math. J. (2) 22 (1970), 325-332.

[13] A. Caldarella and A.M. Pastore, Mixed 3-Sasakian structures and curvature, Ann. Polon. Math. 96 (2009), 107-125.

[14] W.M. Boothby and H.C. Wang, On contact manifolds, Ann. of Math. (2) 68 (1958), 721-734.

[15] Y. Hatakeyama, Some notes on differentiable manifolds with almost contact structures, Tohoku Math. J. (2) 15 (1963), 176-181.

[16] K. Sakamoto, On the topology of quaternion Kähler manifolds, Tohoku Math. J. (2) 26 (1974), 389-405.

[17] M. Konishi, On manifolds with Sasakian 3-structure over quaternion Kaehler manifolds, Kōdai Math. Semin. Rep. 26 (1975), 194-200.

[18] V. Cruceanu, P. Fortuny and P.M Gadea, A survey on paracomplex geometry, Rocky Mt. J. Math. 26 (1996), no.1, 83-115.

[19] S. Kobayashi and K. Nomizu, Foundations of Differential Geometry, 2 volumes, Interscience PUN., New York 1963, 1969.

[20] P. Libermann, Sur les structures presque quaternioniennes de deuxième espèce, C.R. Acad. Sci Paris 234 (1952), 1030-1032.

[21] E. García-Río, Y. Matsushita and R. Vázquez-Lorenzo, Paraquaternionic Kähler manifolds, Rocky Mt. J. Math. 31 (2001), no. 1, 237-260. 
[22] N. Blažić, Para-quaternionic projective spaces and pseudo Riemannian geometry, Publ. Inst. Math. 60(74) (1996), 101-107.

[23] A. Besse, Einstein manifolds, Springer-Verlag, New York, 1987.

[24] K. Sekigawa, Almost Hermitian structures on twistor bundles, Proc. Ramanujan Centennial Int. Conf., Annamalainagar (1987), 127-136.

[25] A. Gray, Curvature identities for Hermitian and almost-Hermitian manifolds, Tohoku Math. J. (2) 28 (1976), 601-612.

[26] M. Brozos-Vázquez, P. Gilkey, S. Nikčević and R. Vázquez-Lorenzo, Geometric Realizations of para-Hermitian curvature models, Results in Mathematics 56 (2009), 319-333.

[27] E. Calvino-Louzao, E. García-Río, P. Gilkey and R. Vázquez-Lorenzo, Higher-dimensional Osserman metrics with non-nilpotent Jacobi operators, Geom. Dedicata 156 (2012), 151-163.

[28] B. Alexandrov, G. Grantcharov and S. Ivanov, Curvature properties of twistor spaces of quaternionic Kähler manifolds, J. Geom. 62 (1998), no. 1-2, 1-12.

[29] S. Sasaki, On differentiable manifolds with certain structures which are closely related to almost contact structure I, Tohoku Math. J. (2) 12 (1960), 459-476.

[30] I. Sato, On a structure similar to the almost contact structure, Tensor, New Ser. 30 (1976), 219-224.

[31] D.E. Blair, Contact manifolds in Riemannian Geometry, Lectures Notes in Math. 509, Springer-Verlag, 1976.

[32] S. Ianuş and G.E. Vîlcu, Some constructions of almost para-hyperhermitian structures on manifolds and tangent bundles, Int. J. Geom. Methods Mod. Phys. 5 (2008), no. 6, 893-903.

[33] S. Ianuş, M. Visinescu and G.E. Vîlcu, Conformal Killing-Yano tensors on manifolds with mixed 3-structures, SIGMA, Symmetry Integrability Geom. Methods Appl. 5 (2009), Paper 022, 12 pages.

[34] A. Caldarella and A.M. Pastore, Mixed metric 3-contact manifolds and paraquaternionic Kähler manifolds, Rapp. Int. 57/2008, Dept. of Math., University of Bari, math$\mathrm{dg} / 0803.2974$.

[35] M. Barros, A. Ferrández, P. Lucas and M. Merono, Willmore tori and Willmore-Chen submanifolds in pseudo-Riemannian spaces, J. Geom. Phys. 28 (1998), no. 1-2, 4566.

[36] O. Dearricott, Positive sectional curvature on 3-Sasakian manifolds, Ann. Global Anal. Geom. 25 (2004), No.1, 59-72.

[37] M. Falcitelli, S. Ianuş and A.M. Pastore, Riemannian submersions and related topics, World Scientific, 2004.

\section{Gabriel Eduard VÎLCU}

Petroleum-Gas University of Ploieşti,

Department of Mathematics and Computer Science,

Bulevardul Bucureşti, Nr. 39, Ploieşti 100680, Romania

E-mail: gvilcu@upg-ploiesti.ro

and

University of Bucharest, Faculty of Mathematics and Computer Science,

Research Center in Geometry, Topology and Algebra

Str. Academiei, Nr. 14, Sector 1, Bucharest 70109, Romania

E-mail: gvilcu@gta.math.unibuc.ro

\section{Rodica Cristina VOICU}

University of Bucharest, Faculty of Mathematics and Computer Science

Research Center in Geometry, Topology and Algebra

Str. Academiei, Nr. 14, Sector 1, Bucharest 72200, Romania

E-mail: rcvoicu@gmail.com 